\title{
Material Properties of GaN in the Context of Electron Devices
}

\author{
H. Morkoç ${ }^{1}$, R. Cingolani ${ }^{1}$, W. Lambrecht ${ }^{2}$, B. Gil ${ }^{3}$, H.-X Jiang ${ }^{4}$, J. Lin $^{4}$, \\ D. Pavlidis ${ }^{5}$, K. Shenai ${ }^{6}$,
}

Cite this article as: MRS Internet J. Nitride Semicond. Res. 4S1, G1.2 (1999)

\begin{abstract}
Wide bandgap nitride semiconductors have recently attracted a great level of attention owing to their direct bandgaps in the visible to ultraviolet regions of the spectrum as emitters and detectors. However, this material system with its favorable heterojunctions and transport properties began to produce very respectable power levels in microwave amplifiers. If and when the breakdown fields achieved experimentally approaches the predicted values, this material system may also be very attractive for switching power devices. In addition to the premature breakdown, a number of scientific challenges remain including a clear experimental investigation of polarization effects. In this paper, transport properties as pertained to electronic devices and potential switching devices, and polarization effects will be treated.
\end{abstract}

\section{Introduction:}

Gallium nitride and its alloys with InN and AIN are important semiconductor materials with applications to emitters and detectors (visible to UV range) and high power/temperature electronics. ${ }^{1.23 .45 .6}$ The production of commercial blue, green and yellow nitride LEDs, demonstration of $\mathrm{CW}$ injection lasers and $\mathrm{UV}$ detectors are truly extraordinary. ${ }^{7.89}$ The large intrinsic dielectric breakdown fields, good thermal conductivity, and favorable transport properties make nitride semiconductors desirable for high power electronic devices. ${ }^{10}$ For example, modulation doped FETs with a record power density of $6 \mathrm{~W} / \mathrm{mm}$ have been reported at $10 \mathrm{GHz} . "$

Being non-centro-symmetric, nitrides exhibit large piezoelectric effects when under stress along the $c$ direction. What is not as appreciated is the spontaneous polarization at heterointerfaces caused by the ionicity and uniaxial nature of the wurtzite. Polarization causes a sizable red shift (Stark Effect) in transition energies in InGaN/GaN ${ }^{12.13}$ and $\mathrm{AlGaN} / \mathrm{GaN}^{14.15 .16 .17}$ quantum wells. Polarization and pyroelectric effects due to heterointerfaces in an ionic crystal, misfit and thermal strain, anisotropy, and temperature gradients have important ramifications in electronic devices,

1. Virginia Commonwealth University, P.O. Box 843072, Richmond, USA 23284-3072

* Permanent address: Dept. Material Science, University of Lecce, Lecce Italy

2. Department of Physics, Case Western Reserve University, Cleveland, $\mathrm{OH} 44106$

3. Montpellier University II, 34095 Montpellier CEDEX 5 France

4. Kansas State University, Manhattan, Kansas 66506-2601

5. University of Michigan, Ann Arbor, MI 48109-2122

6. University of Illinois, Chicago, IL 60607-7053 
particulary in modulation doped FETs. For example, electric field caused by polarization effects can increase or decrease interfacial free carrier concentrations. As in the case of quantum wells, the literature interpretation of polarization effects in devices has so far been lumped into piezoelectric effects. ${ }^{18,19}$.

Despite highly imperfect material, device performance in both emitters/detectors, and the microwave amplifiers has been truly outstanding. If the defects causing premature breakdown were reduced/eliminated, one would wonder whether it would be wrong at all to attempt to develop power/switching devices with large hold voltages and current handling capabilities. In this paper, performance advantages of nitrides in vertical high power devices will be visited along with the topical topic of polarization issues.

\section{Transport Properties as Pertained to Electronic Devices}

Transport properties of, namely n-type, $\mathrm{GaN}$ and to some extent its related binaries and alloys have been calculated and experimentally investigated. There are a few noteworthy features, among which are the high peak and saturation electron velocity in $\mathrm{GaN}$ that does not degrade with doping, and temperature nearly as much when compared to conventional compound semiconductors. Likewise, the low field mobility does not degrade with field as fast due to the large LO phonon energy. Very intriguing are the measured low field mobilities in modulation doped structures which are nearly twice as large as the predicted polar optical phonon limited mobility at room temperature. The optimistic view could be that certain scattering mechanisms are mitigated in such structures and that the bulk low field mobility in GaN at room temperature could be as high as some $2000 \mathrm{~cm}^{2} \mathrm{~V}^{-1} \mathrm{~s}^{-1}$. If so, this would pave the way for much coveted very low power loss in $\mathrm{GaN}$ based amplifiers and switches. Also important are transport properties at low temperatures. The low temperature electron mobility in modulation doped structures is above $7,000 \mathrm{~cm}^{2} \mathrm{~V}^{-1} \mathrm{~s}^{-1} \mathrm{~s}$, and the electron velocity increases which provide basis for a compelling argument that cooled $\mathrm{GaN}$ based devices utilizing electron transport should be capable of offering much enhanced performance. Whether to cool or not to cool a given semiconductor device must be decided following an overall power/size optimization scheme of all the components that include the power supplies, supporting circuitry, and heat dissipation related hardware. Such optimization schemes often lead to suggestions that the system should be cooled to a certain temperature which bode well for GaN based modulation doped FETs.

Figures of merit for general power switching applications have been examined and it has been shown that power devices in wide energy bandgap materials provide several orders of magnitude improvement in power-handling capability ${ }^{20}$. Compared to $\mathrm{Si}$, calculations for ideal $\mathrm{GaN}$ show that GaN offers more than two orders of magnitude improvement in electrical conductivity per unit area $\left(\sigma_{\mathrm{A}}\right)$, and nearly a factor of 10 improvement in maximum power switching frequency $\left(f_{\max }\right)$. Calculations have also been performed to determine thermal runaway limitations and results show that a $\mathrm{SiC}$ or a GaN PN junction diode can sustain $6 \mathrm{X}$ higher junction temperature rise compared to a silicon diode before catastrophic burnout occurs which shows potential for device operation above $600^{\circ} \mathrm{C}$. Calculations have been performed to estimate performance improvements for $\mathrm{SiC}$ and $\mathrm{GaN}$ Schottky rectifiers. These results indicate that nearly a five-fold reduction in $\mathrm{V}_{\mathrm{F}}$ can be expected for $200-\mathrm{V} \mathrm{SiC}$ and GaN Schottky rectifiers operating at a current 
density of $100 \mathrm{~A} / \mathrm{cm}^{2}$. Above $200 \mathrm{~V}$, silicon Schottky rectifiers are impractical because of high $\mathrm{V}_{\mathrm{F}}$ and increased surface leakage currents. These results suggest the feasibility of fabricating high performance $\mathrm{SiC}$ and $\mathrm{GaN}$ Schottky rectifiers with $\mathrm{V}_{\mathrm{BD}}>5000 \mathrm{~V}$. In addition, for identical current-handling capability, $\mathrm{SiC}$ and $\mathrm{GaN}$ devices are expected to be an order of magnitude smaller in die size, thus offsetting any increase in wafer processing cost.

Heterojunction bipolar transistors based on nitride semiconductors are beginning to receive a good deal of attention for high power/switching applications. Arguments used above for rectifiers also apply for HBTs as the requirements for the collector region of an HBT are similar to those for rectifiers and switches. For example, for a given breakdown voltage, the GaN drift region can be either much thinner and/or doped much higher as compared to other semiconductors with smaller breakdown strengths (about a factor 30 compared to $\mathrm{Si}$ ). This would result in low on resistances, and shorter transit times (nearly by two orders of magnitude as compared to $\mathrm{Si}$ ), both of which are very much desirable. However, the caveat here is that GaN and related semiconductors have not yet demonstrates their intrinsic breakdown strengths, exhibiting instead premature breakdown. Here as well as above, the discussion is based on intrinsic breakdown strengths which is justifiable for prediction purposes and expecting natural advances in the technology of these materials that occur with time.

\section{Polarization Effects}

As alluded to earlier, observations support the presence of electric field in GaN-based heterostructures. The genesis is two fold: piezoelectric effects and the difference in spontaneous polarization between $\mathrm{AlGaN}$ and $\mathrm{GaN}$ even in the absence of strain. Spontaneous polarization was only recently fully understood, see King-Smith and Vanderbilt ${ }^{21}$, Resta $^{22}$, and Bernardini and Fiorentini ${ }^{23}$. Nitrides lack inversion symmetry and exhibit piezoelectric effects when strained along [0001]. Piezoelectric coefficients are almost an order of magnitude larger than in traditional III-V, see Ref. 24, as shown in the table below. In addition, wurtzite GaN has a unique axis, thus allowing spontaneous polarization $\left(\mathbf{P}_{\mathbf{0}}\right.$ whose values are given below) even in the absence of strain. This manifests itself as polarization charge at hetero-interfaces.

$\begin{array}{llll} & \text { AlN } & \text { GaN } & \text { InN } \\ \mathrm{e}_{33}\left(\mathrm{C} / \mathrm{m}^{2}\right) & 1.46 & 0.73 & 0.97 \\ \mathrm{e}_{31}\left(\mathrm{C} / \mathrm{m}^{2}\right) & -0.60 & -0.49 & -0.57 \\ \mathbf{P}_{0}\left(\mathrm{C} / \mathrm{m}^{2}\right) & -0.081 & -0.029 & -0.032 \\ \left\{\mathrm{e}_{31}-\left(\mathrm{C}_{13} / \mathrm{C}_{33}\right) \mathrm{e}_{33}\right]\left(\mathrm{C} / \mathrm{m}^{2}\right) & -0.86 & -0.68 & -0.90\end{array}$

Note that these are all in $\mathrm{C} / \mathrm{m}^{2}$ and that $1 \mathrm{C} / \mathrm{m}^{2}=6.25 * 10^{14} \mathrm{e} / \mathrm{cm}^{2}$

Let us compare the relative importance of spontaneous polarization to piezoelectric polarization. For a biaxially strained layer the effective piezoelectric polarization is given by

$$
\mathrm{P}_{r}^{\text {prero }}=\left[\mathrm{e}_{31}-\left(\mathrm{C}_{31} / \mathrm{C}_{33}\right) \mathrm{e}_{33}\right] \varepsilon_{\perp}
$$


Where $\varepsilon_{\perp}=\varepsilon_{x x}+\varepsilon_{y y}$ is the in-plane strain and $C_{31}$ and $C_{33}$ are elastic constants. The numerical values for $\left[\mathrm{e}_{31}-\left(\mathrm{C}_{31} / \mathrm{C}_{33}\right) \mathrm{e}_{33}\right]$ are listed in the above table using the values for elastic constants from Kim et al. $^{25}$ and piezoelectric and spontaneous polarization data from Bernardini et al. For $\mathrm{Al}_{\mathrm{x}} \mathrm{Ga}_{1-\mathrm{x}} \mathrm{N}$ pseudomorphically strained on a relaxed GaN substrate, the strain $\varepsilon_{\perp}$ is expected to be proportional to $\mathrm{x}$ and given by $\varepsilon_{\perp}=2 \mathrm{x}\left(\mathrm{a}_{\mathrm{GaN}}-\mathrm{a}_{\mathrm{AIGaN}}\right) / \mathrm{a}_{\mathrm{AIGaN}}$, which is $0.0495 x$ (using $3.112 \AA$ for $\mathrm{AlN}$ c-plane lattice constant and $3.189 \AA$ for $\mathrm{GaN}$ c-plane lattice constant, here $x$ is in the range of 0 1 and depicts the AlN mole fraction in the alloy) and is tensile. The piezoelectric polarization is then $\mathrm{P}^{\text {piezo }}=-4.26 x * 10^{-2} \mathrm{C} / \mathrm{cm}^{2}$ or $2.66 \mathrm{x} * 10^{1.3} \mathrm{e} / \mathrm{cm}^{2}$ and points in the [0001] direction. The corresponding difference in spontaneous polarization between $\mathrm{Al}_{x} \mathrm{Ga}_{1 \cdot x} \mathrm{~N}$ and $\mathrm{GaN}$ is also expected to be proportional to $\mathrm{x}$ and is given by $\Delta \mathrm{P}^{\mathrm{spon}}=-0.052 x\left(\mathrm{C} / \mathrm{m}^{2}\right)$ or $3.25 \mathrm{x} * 10^{1.3} \mathrm{e} / \mathrm{cm}^{2}$. Note that the two polarization effects have the same sign for Ga polarity and tensile strain in the alloy and point in the [0001] direction. For an $\operatorname{In}_{x} \mathrm{Ga}_{1-x} \mathrm{~N}$ layer, the situation is rather different in that the differential spontaneous polarization between $\operatorname{In}_{x} \mathrm{Ga}_{1-x} \mathrm{~N}$ and $\mathrm{GaN}$ is much smaller, $\Delta \mathrm{P}^{\mathrm{spon}}=-0.003 x$ which translates to $1.88 \mathrm{x} * 10^{12} \mathrm{e} / \mathrm{cm}^{2}$. Furthermore, the $\operatorname{In}_{\mathrm{x}} \mathrm{Ga}_{1 \cdot \mathrm{x}} \mathrm{N}$ layer on $\mathrm{GaN}$ would be under compressive stress $\varepsilon_{\perp}=-0.195 x$ using $3.533 \AA$ and $3.189 \AA$ for c-plane lattice constants for InN and GaN. Here $x$ is in the range of $0-1$ and depicts the InN mole fraction, and $\mathrm{P}^{\text {pizzo }}=+0.176 x$ or $1.1 x^{*} 10^{14} \mathrm{e} / \mathrm{cm}^{2}$. For an InN mole fraction of $0.15, \mathrm{P}^{\text {piezo }}=1.65 * 10^{13} \mathrm{e} / \mathrm{cm}^{2}$. Here the piezoelectric polarization dominates and is opposite in direction but even larger in absolute magnitude. In the AlGaN case, the sign of the polarization is such that it produces a potential energy for electrons sloping down from the Ga face towards the $\mathrm{N}$ face. Here $\mathrm{Ga}$ and $\mathrm{N}$ faces correspond to polarities where the bonds (single) along the c-directions are from Ga cations to $\mathrm{N}$ anions, and $\mathrm{N}$ anions to $\mathrm{Ga}$ cations, respectively.

For a discussion of polarity as opposed to surface termination and definitions of $\mathrm{Ga}$ face, $\mathrm{N}$ face, see Hellman ${ }^{26}$. Thus for a structure with Ga polarity, the potential will slope down from the $\mathrm{AlGaN}$ surface towards the $\mathrm{AlGaN} / \mathrm{GaN}$ interface and will help to drive free electrons towards the interface forming a 2DEG For example, if there is an Ohmic metal contact on the AlGaN surface, electrons will flow towards the 2DEG below that layer. The most favorable situation for enhancing sheet carrier concentration would occur for an InGaN (under compressive strain) quantum well on top of a relaxed n-GaN and below a AlGaN barrier (under tensile strain) with the entire structure having cation $(\mathrm{Ga})$ polarity. In that case, the field will slope down towards the InGaN/AlGaN interface in the quantum well and will help localize the carriers in the 2DEG. Note that the piezoelectric polarizations estimated here are based on the theoretical values for perfectly insulating material. The field will be screened by the carriers present in each layer. For example, if carriers flow from a metal contact towards the 2DEG, then this will set up a counteracting field. The equilibrium self-consistent field is ultimately determined by the requirement that the chemical potential for electrons (i.e. the Fermi level) must be constant throughout the structure and thus depends on the doping and band bending in the substrate and possibly in each of the layers. At the least, one may expect these fields will be reduced by a factor corresponding to the macroscopic dielectric constant, i.e. a factor of order 10 but possibly larger if the layers acquire conductivity by free carriers. So, a more realistic expectation for the effects on sheet carrier concentration is of order $10^{11}-10^{12} \mathrm{e} / \mathrm{cm}^{2}$. 
In traditional device structures lacking polarization effects and for a uniform dopant concentration one obtains parabolically varying potentials with distance, whereas here there are linear terms due to the polarization on top of the parabolic terms. These linear terms lead to variations of the potential over a shorter distance scale determined by the thickness of the layers whereas the parabolic terms correspond to the space-charge regions. Thus the linear terms may help to localize carriers if the polarity of the structure is chosen properly. Some further words of caution about the above estimates are needed. If the $\mathrm{AlGaN}$ layers are not pseudomorphic but partially relaxed (by misfit dislocations for example), then the piezoelectric effect would be reduced but the spontaneous polarization would not. If the interfaces are not atomically sharp but exhibit a certain degree of interdiffusion then the differences in spontaneous polarization would be reduced as well if such grading occurs over a large thickness. Finally, if domains with inverted polarity exist then the overall polarization effects may be washed out. Also note that in a inverted structure with $\mathrm{N}$ polarity towards the surface, it may be possible to create a 2DHG (holegas) at the $\mathrm{AlGaN} / \mathrm{substrate} \mathrm{GaN}$ interface, but if a n-type GaN layer is placed on top, a 2DEG may form on top of the AlGaN layer.

Anisotropy in wide bandgap semiconductors is expected to cause thermal stimulus and thermal gradients. The latter is quite likely in devices where the junction temperatures are high against a relatively cool bulk. In such a case, the thermally induced electric field, pyroelectric effect, would be present with effects similar to those described above. ${ }^{27}$ This phenomenon has not been studied in detail in nitride semiconductors. Contrary to this lack of attention, the electric field generated by thermal gradients, occurs readily in power devices, is comparable to the fields caused by applied voltages in nitride based FET like devices.

\section{Polarization Effects in Device Like-Structures}

While physics appears to be converging, reports on FETs appear to suggest that free carriers can be provided by polarization and that the experimental results agree with theory ${ }^{28.29}$ Results obtained in our laboratory, in conjunction with band discontinuities, with respect to polarization effects point to the importance of spontaneous polarization ordering of the barrier layer with respect to the smaller bandgap material, and polarization in general. ${ }^{30.31 .32}$ The polarization charge can be screened by to weakly bound and free carriers present in the system which may aid or hamper device effort. The complicating factor in nitrides is the lack of polarity control, Ga or $\mathrm{N}$ polarity, and lack of uniform polarity meaning presence of inversion domains.

As a result of polarization, the static potential at the GaN/AIN interface is different from that at the $\mathrm{AlN} / \mathrm{GaN}$ interface giving rise to interface charge larger than the charge densities used in devices. A substantial level of effort has been expended toward determining band discontinuities, but the field is in desperate need of more in depth investigations in improved structures. The observed asymmetry in AIN/GaN and GaN/AlN interfaces caused by spontaneous polarization is within the experimental errors of Martin et al. Inversion domains combined with any strain in nitride based films lead to flipping PE fields with untold adverse effects on our ability to characterize the films let alone exploit this phenomenon for devices. Such flipping field would also cause much increased scattering of carrier as they traverse in the c-plane. Simply put, identical device structures with different polarity layers would have widely differing performance underscoring the importance that these issues will have to be investigated and reconciled. The 
polarity mixing from one domain to the other causes the PE induced electric field to flip, causing a variation in the sheet carrier concentration along the channel of an FET like device. The same polarity mixing would have deleterious effects in the base of an HBT as well and depending on the polarity the induced field would either aid or impede minority carrier transit.

Polarization effects manifest themselves in quantum wells as Stark effect. Time resolved photoluminescence measurements performed in GaN/AlGaN MQWs indicated that temporal evolution of the A exciton peak undergoes a red shift as the recombination depletes the excess carriers. If one assumes that the red shift is due to polarization induced field, one can determine the intensity of the field. The picture may be more complex and or suffer from variation in samples as blue shift too has been observed which may have to do with interwell excitons and oblique excitons. ${ }^{33}$ Though controversial, in lasers the injected carrier concentrations even at transparency are comparable to polarization induced charge, $10^{13} \mathrm{~cm}^{-2}$, which means the PE induced field is screened. However, the PE effect reduces the gain at lower injection levels due, among others, to reduced oscillator strength.

Recent experimental results support that the built-in field originates primarily from the spontaneous polarization charge formed at the GaN/AlGaN interfaces with a minor contribution from the piezoelectric field induced by the strain (either lattice mismatch and thermal strain) as has been predicted. The experiments reveal that the fundamental transition of the quantum wells occurs at an energy well below the bulk GaN gap for well width larger than $3 \mathrm{~nm}$ (for the specific case of $\mathrm{GaN} / \mathrm{Al}_{0.15} \mathrm{Ga}_{0.85} \mathrm{~N}$ ). Care was taken to assure that the transitions observed are intrinsic origin by performing two-photon absorption experiments with approximately half-gap excitation $\left(2 \hbar \omega \approx \mathrm{E}_{\text {lelh }}\right.$ ) and observing that linear and non-linear PL lead to same transition energies. The total polarization charge can be written as $\mathrm{P}_{\text {tol }}=\mathrm{P}_{\text {piezo }}+\mathrm{P}_{\text {spont }}$, where $\mathrm{P}_{\text {piezo }}$ is the piezoelectric charge caused by the lattice mismatch $(\mathrm{lm})$ strain and by the thermal strain $(\mathrm{ts})\left[\mathrm{P}_{\text {piezo }}=\mathrm{P}_{\mathrm{Im}}+\mathrm{P}_{\mathrm{ts}}\right]$, whereas $P_{\text {spont }}$ represents from the spontaneous polarizability of the GaN/AlGaN interface, as clearly demonstrated by the recent works of Bernardini et al. For an alternating sequence of wells $(w)$ and barriers (b) the total electric field in the well can be calculated as

$$
\begin{aligned}
& \mathrm{F}_{\mathrm{w}}=-4 \pi \mathrm{L}_{\mathrm{b}}\left(\mathrm{P}_{\text {tot }}^{\mathrm{w}}-\mathrm{P}_{\text {tot }}^{\mathrm{b}}\right) /\left(\mathrm{L}_{\mathrm{w}} \varepsilon_{\mathrm{b}}+\mathrm{L}_{\mathrm{b}} \varepsilon_{\mathrm{w}}\right) \\
& \mathrm{F}_{\mathrm{b}}=-4 \pi \mathrm{L}_{\mathrm{w}}\left(\mathrm{P}_{\text {tot }}^{\mathrm{b}}-\mathrm{P}_{\text {tot }}^{\mathrm{w}}\right) /\left(\mathrm{L}_{\mathrm{b}} \varepsilon_{\mathrm{w}}+\mathrm{L}_{\mathrm{w}} \varepsilon_{\mathrm{b}}\right)
\end{aligned}
$$

Where $\varepsilon$ is the dielectric constant of the particular layer. The piezoelectric charge induced by the in-plane lattice mismatch can be found from Eq. 1 using the in-plane strain for AlGaN/GaN as $\varepsilon_{\perp}=2 \times\left(a_{G a N}-a_{A I G a N}\right) / a_{A I G a N}$ where the $\mathrm{AlGaN}$ is pseudomorphically strained on a relaxed $\mathrm{GaN}$ substrate. Thick GaN buffer layers ensures that the $\mathrm{GaN}$ quantum wells are not under misfit strain. Moreover, since the $\mathrm{Al}_{0.15} \mathrm{Ga}_{0.85} \mathrm{~N}$ layers are thin and grow pseudomorphically, ${ }^{34}$ they undergo a tensile in-plane strain $\varepsilon_{\perp}=0.742 \%$. This results in a piezoelectric polarization charge $\mathrm{P}_{\mathrm{lm}}^{\mathrm{w}}=0$ in the wells and $\mathrm{P}_{\mathrm{lm}}^{\mathrm{b}}=-3.99 * 10^{12} \mathrm{ecm}^{-2}$ in the $\mathrm{Al}_{0.15} \mathrm{Ga}_{0.85} \mathrm{~N}$ barriers. The thermal strain amounts to some $0.03 \%$, resulting in an additional polarization charge of the order of $\mathrm{P}^{\mathrm{w}}{ }_{\mathrm{ts}}=+$ 
$2.57 * 10^{11} \mathrm{ecm}^{-2}$. For spontaneous polarization, we take the recent data of Bernardini et al. leading to $\mathrm{P}_{\mathrm{sp}}^{\mathrm{u}}=-1.81 * 10^{12} \mathrm{ecm}^{-2}$ and $\mathrm{P}_{\mathrm{sp}}^{\mathrm{b}}=-7.64 * 10^{12} \mathrm{ecm}^{-2}$, the latter value being obtained by linear interpolation of the GaN and AlN values $\left(\mathrm{P}_{\mathrm{sp}}=-5.09 * 10^{13} \mathrm{ecm}^{-2}\right.$ in AlN). The diffrential spontanous polarization between the $\mathrm{Al}_{0,15} \mathrm{Ga}_{0.85} \mathrm{~N}$ barrier and the $\mathrm{GaN}$ well is then $-5.83 * 10^{12}$ $\mathrm{ecm}^{-2}$. By using these data and eq.(2) one can calculate the built-in field in the different samples, which turns out to vary in the range 1-3 MV/cm depending on the actual well width. Neglecting high-field effects and corrections for self-consistency, the red-shift caused by the built-in field in the wells is given by the quadratic Stark effect. ${ }^{35.36}$

The measured ground level transition energies and those calculated in the square well approximation with inclusion of the Stark shift are in very good agreement. The agreement between theoretical and experimental data is very reasonable, especially if we take into account that the nominal growth parameters were used in the calculations, without any fitting parameter.

The agreement between the calculated and measured data can in fact be substantially improved by the use of a self-consistent tight-binding (TB) model. ${ }^{37}$ The tight-binding model is used to describe the electronic structure in the entire Brillouin zone, up to several $\mathrm{eV}$ above the fundamental gap, thus overcoming the well-known limitations of the envelope function approach. For the specific case of nitride semiconductors, the parameters of our empirical TB model were determined by fitting the band-structure of ref. 38 . For the self-consistent calculations the electron and hole quasi-Fermi levels are calculated for a given photo-injected charge density. The resulting electron and hole distribution functions ( $n$ and $p$, respectively) are used to solve the Poisson equation. The Poisson equation is solved assuming zero-field at the boundaries. The obtained potential is thus inserted into the TB Schroedinger equation that is solved to get the energies and wavefunctions. The new quasi-Fermi levels are thus recalculated and the procedure is iterated until self-consistency is achieved. The agreement between the selfconsistent calculations and the experimental data is very good both in terms of the trend and absolute values.

In conclusions, $\mathrm{GaN}$ and related materials exhibit transport characteristics that are very well suited for electronic devices based on electron transport as evidenced by the recent high power MODFETs with record power performance supplanted all the other competing semiconductors at microwave frequencies. Assumes that some day the intrinsic dielectric breakdown strength can be achieved, applicable figures of merit for switching/power device indicate that nitrides would be equally successful. Finally, recent theoretical and experimental investigations indicate that polarization induced charge is important in nitride semiconductors with device implications and that spontaneous polarization is dominant over strain induced piezoelectric polarization in typical $\mathrm{GaN} / \mathrm{AlGaN}$. This dominance would increase if the $\mathrm{AlGaN}$ grown on GaN relaxes in full or in part. However, the differential spontaneous polarization between $\mathrm{GaN}$ and $\mathrm{InN}$ is much smaller than that of GaN/AlN interfaces. Assuming that the InGaN on GaN is fully strained, the piezoelectric component of polarization would dominate. Full or partial relaxation of InGaN on $\mathrm{GaN}$ due to defects would reduce this polarization as well. For now, optical transitions in $\mathrm{GaN} / \mathrm{AlGaN}$ quantum wells investigated support the assertion that spontaneous polarization is dominant and must not be lumped with piezoelectric polarization. 
Acknowledgments: One of the authors, R.C. gratefully acknowledges Virginia Commonwealth University for hospitality. The work at Lecce is partially sponsored by INFM-Italia through Progetto Sud, and that at VCU by the Air Force Office of Scientific Research and Office of Naval Research under the direction of Drs. G. L. Witt, C.E. C. Wood, Y. S. Park and Mr. M. Yoder.

\section{References:}

S.T. Strite and H. Morkoç, J. Vac. Sci. and Tech. B10, pp.1237-1266 (1992).

2 H. Morkoç, S. Strite, G. B. Gao, M.E. Lin, B. Sverdlov, and M. Burns, J. Appl. Phys. Rev. 76, pp. $1363-$ 1398 (1994).

3 S. N. Mohammad and H. Morkoç, Progress and Prospects of Group III-V Nitride Semiconductors, Progress in Quantum Electronics, Vol. 20, No. 5/6, pp. 361-525, (1996).

4 Group IIl Nitride Semiconductor Compounds, Edited by B.Gil, Clarendon Press, Oxford (1998), ISBN 0-19-850159-5.

5 H. Morkoç, Wide Bandgap Nitride Devices, Springer, in press.

6 S. Nakamura and G. Fosol, The Blue Laser, Springer Verlag, 1997.

7 M. Razeghi and A. Rogalski, J. Appl. Phy. 79, 7433 (1996).

${ }^{8}$ G. Y. Xu, A. Salvador, W. Kim, Z. Fan, C. Lu, H. Tang, H. Morkoç, G. Smith, M. Estes, B. Goldenberg, W. Yang, and S. Krishnankutty, Appl. Phys. Letts. 71, pp. 2154-2156 (1997).

9 H. Morkoç, îWurtzite GaN-Based Heterostructures by Molecular Beam Epitaxy, IEEE J.Selected topics in Quantum Electronics, Eds. Richard Miles and I. Akasaki, Vol. 4, Number 3, pp. 537-549 (1998).

${ }^{10} \mathrm{H}$. Morkoç, iBeyond SiC III-V Nitride Based Heterostructures and Devices, in SiC Materials and Devices, Ed. Y. S. Park, Vol. 52, pp. 307-394, Academic Press (1998).

"S. T. Sheppard et al. presented at Device Research Conference, June 1998, Charlottesville, VA. Other GaN MODFETs references include: Y.-F. Wu, IEEE Electron Device Letters 19, pp 50-53 (1998); A.T. Ping et al., IEEE Electron Device Letters 19, pp 54-56 (1998).

12 T.Takeuchi, C.Wetzel, S.Yamaguchi, H.Sakai, H.Amano, I.Akasaki, Y.Kaneko, S.Nakagawa, Y.Yamaoka, and N.Yamada, Appl.Phys. Lett. 73, 1691 (1998)

${ }^{13}$. J.S.Im, H.Kollmer, J.Off, A.Sohmer, F.Scholz, and A.Hangleiter, Phys. Rev. B57, R9435 (1998)

${ }^{14}$. H. S. Kim, J. Y. Lin, H. X. Jiang, W.W. Chow, A. E. Botchkarev, and H. Morkoç, , Appl. Phys. Letts. In press.

${ }^{15}$. M. Leroux, N. Grandjean, J. Massies, B. Gil, P. Lefebvre, and P. Bigenwald, Phys. Rev. B. in press;

16. Roberto Cingolani, A. Botchkarev, H. Tang, Hadis Morkoç, Giuliano Colì̀ and Mauro Lomascolo, A. Di Carlo, and P.Lugli, Appl. Phys. Letts,.submitted.

${ }^{17}$. A. Bykhovski, B. Gelmont, and M. Shur, Appl. Phys. Lett. 63, 2243, (1993).

18 . E.T. Yu, G.J. Sullivan, P.M. Asbeck, C.D. Wang, D. Qiao, and S.S.Lau, Appl. Phys. Lett. 71, 2794 (1997)

19 . P.M. Asbeck, E.T. Yu, S.S. Lau, G.J. Sullivan, J. Van Hove and J. Redwing, Elec. Lett. 33(14), 1230 (1997). 
2t. K. Shenai, R. S. Scott, and B. J. Baliga, IEEE Trans. Electron Devices, vol. 36, no. 9, pp.1811-1823, Sept. 1989.

2. R.D. King-Smith and D. Vanderbilt, Phys. Rev. B 47, 1651 (1990).

22 . R. Resta, Rev. Mod. Phys. 66, 899 (1994),

${ }^{23}$. F. Bernardini and V. Fiorentini, Phys. Rev. B. 57, No 16, pp.1-4, 15 April (1998)

${ }^{24}$. F. Bernardini, V. Fiorentini, and D. Vanderbilt, Phys. Rev. B 56, R 10024 (1997)

${ }^{25}$. K. Kim, W. R. L. Lambrecht, and B. Segall Phys. Rev. B 56, 7018 (1997)

${ }^{26}$. E.S. Hellman, in MRS Internet J. Nitride Semiconductor Research 3, 11.

${ }^{27}$. A.D. Bykhovski, V. V. Kaminski, M. S. Shur, Q. C. Chen, and M. A. Khan, Appl. Phys. Lett., 69,3254, (1996).

2\% . P.M. Asbeck, E.T. Yu, S.S. Lau, G.J. Sullivan, J. Van Hove and J. Redwing, Electronics Lett. 33(14), 1230 (1997).

29 . E.T. Yu, G.J. Sullivan, P.M. Asbeck, C.D. Wang, D. Qiao and S.S. Lau, Appl. Phys. Lett. 71, 2794 (1997

*) . G. A. Martin, S. Strite, A. Botchkarev, A. Agarwal, A. Rockett, H. Morko•, W. R. L. Lambrecht and B. Segall, Appl. Phys. Lett. 65, 610 (1994).

${ }^{31}$. G. A. Martin, PhD. Thesis, ÒSemiconductor electronic band alignment at heterojunctions of wurtzite AlN, GaN and InNÓ, Department of Physics, University of Illinois, 1996.

32 .G. A. Martin, A. Botchkarev, A. Agarwal, A. Rockett, H. Morko•, Appl. Phys. Lett. Vol. 68, 2541, (1996).

. B. Gil, P. Lefebvre, J. Allègre, H. Mathieu, N. Grandjean, M. Leroux, J. Massies, P. Bigenwald, and P. Christol, Phys. Rev. B. in press.

${ }^{4}$. T.Takeuchi, C.Wetzel, S.Yamaguchi, H.Sakai, H.Amano, I.Akasaki, Y.Kaneko, S.Nakagawa, Y.Yamaoka, and N.Yamada, Appl.Phys. Lett. 73, 1691 (1998)

"5. G.Bastard, in ÒWave Mechanics Applied to Semiconductor HeterostructuresÓ, Edition de Physique, Paris , France, 1987

36. J.Singh, in "Semiconductor OptoelectronicsÓ (1995)

37. F.Della Sala, A.DiCarlo, P.Lugli, F.Bernardini, V.Fiorentini, R.Scholz, and J.M.Jancu, Appl. Phys. Lett., in press (1998)

3* . J.M.Jancu, R.Scholz, F.Beltram, and F.Bassani, Phys. Rev. B57, 6493 (1995). 